\title{
Personnel rostering: models and algorithms for scheduling, rescheduling and ensuring robustness
}

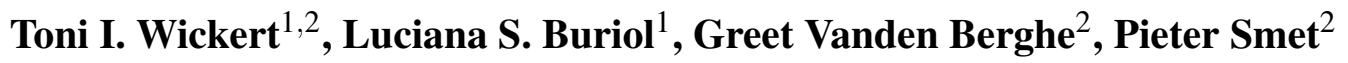 \\ ${ }^{1}$ Institute of Informatics, Federal University of Rio Grande do Sul (UFRGS) \\ 91501-970 - Porto Alegre - Brazil \\ ${ }^{2}$ Department of Computer Science, CODeS - KU Leuven \\ Gebroeders De Smetstraat 1 - 9000 - Gent - Belgium \\ \{tiwickert, buriol\}@inf.ufrgs.br, \{greet.vandenberghe, pieter.smet\}@cs.kuleuven.be
}

\begin{abstract}
Personnel rostering is the process of assigning employees to shifts while respecting a set of constraints. Despite progress in recent decades concerning computation techniques, a considerable number of organizations continue to organize their rosters manually. This thesis seeks to bridge the gap between academia and practice by (i) introducing an integer programming model based on a real-world demand and a matheuristic to quickly generate results, (ii) producing state-of-the-art results for cyclic rostering problems using instances available in the literature, (iii) designing new rerostering strategies for repairing disruptions in multi-skilled rostering scenarios, and (iv) introducing a metric for quantifying and enforcing robustness in rosters. Besides the scientific contributions resulted from the thesis, this work is also relevant in practice. Since April of 2019, Hospital de Clínicas de Porto Alegre employs this PhD's outcome, named ProScheduleSolver to compute its physicians' rosters. The automation takes a few minutes, as opposed to multiple hours in the past, and results in $24,7 \%$ less overtime for the physicians.
\end{abstract}

\section{Introduction}

Personnel rostering has received considerable academic attention in recent decades due to its practical relevance [Ernst et al. 2004]. Hospitals [Burke et al. 2004, Erhard et al. 2018], public transport [Xie and Suhl 2015] and airlines companies [Sohoni et al. 2006] are examples of workplace environments where staff rostering plays a crucial role. The objective in such environments is generally to find schedules which minimize overall cost, while respecting a set of hard constraints. Table 1 presents an example of a roster with nine employees and a planning horizon of one week. Shifts are categorized as Early (E), Late (L) or Night (N), while dashes represent days off.

Despite these significant publications and the existence of solving methods which generate excellent results in limited computation time, the existing literature lacks methods which can be used in real-world scenarios. This not only demands considerable effort on the part of human planners but, in many cases, the rosters generated are of poor quality. This thesis contributes solving methods by investigating several rostering scenarios considering a range of rostering problems. These include rostering (normal and cyclic), rerostering and robust rostering. Problems such as the rerostering feasibility and cyclic staff rostering were proven NP-complete by [Moz and Pato 2007] and [J. Bartholdi 1981], respectively. 
Table 1. Example of a roster with nine employees over a seven day period.

\begin{tabular}{c|ccccccc}
\hline Employee & Mon & Tue & Wed & Thu & Fri & Sat & Sun \\
\hline E1 & - & E & E & E & E & E & N \\
E2 & N & - & - & E & E & E & E \\
E3 & E & N & N & - & - & L & L \\
E4 & L & L & L & N & N & - & - \\
E5 & - & L & L & E & E & N & N \\
E6 & N & - & - & L & L & E & E \\
E7 & - & - & - & L & L & L & L \\
E8 & L & N & N & - & - & - & - \\
E9 & E & E & E & N & N & N & - \\
\hline
\end{tabular}

\section{Contributions of the thesis}

This joint PhD (cotutela) was developed from March of 2015 to November of 2019. The two first years and the last year of the research were conducted at UFRGS and supervised by Luciana $\mathrm{S}$. Buriol. The remaining eighteen months of the $\mathrm{PhD}$ period took place at KU Leuven (Belgium) and was supervised by Greet Vanden Berghe and co-supervised by Pieter Smet. The private defense took place in Gent (Belgium) at KU Lueven on the 21st of August 2019, while the public defense was held in Porto Alegre (Brazil) at UFRGS on the 25 th of November 2019. The thesis was approved with distinction and praise vote (voto de louvor) by all the members of the jury committee.

\subsection{Physician rostering}

The general physician rostering problem (PRP) aims to assign physicians to shifts for each day during a scheduling horizon. The objective is to minimize the cost associated with the violation of soft constraints such as the maximum number of consecutive working days, overtime and physician preferences. The case-specific PRP addressed in the present thesis also includes the concept of locations, meaning that physicians may be allowed to work at specific locations and not at others within the hospital. In addition to minimizing the overall cost and violation of physician preferences, this study also introduces constraints to generate a fair distribution of working hours between those physicians [Wickert et al. 2020].

Hospital de Clínicas de Porto Alegre (HCPA) is located in the south of Brazil and motivated the study regarding physician rostering. The hospital's managers reported a series of problems occurring with their manual scheduling process. These include the long time required to organize and check the accuracy of the rosters, elevated overtime as well as an inefficient balancing of working hours both in terms of overtime and working hours during non-business days across their physicians. In addition, improper rostering decisions due to not consulting the data from previous rostering periods were often incurred.

These circumstances motivated the investigation of existing literature concerning PRPs as well as the nurse rostering problem, given its similarities. The absence of a mathematical formulation that covers all the constraints indicated as important in practice by hospital managers motivated the development of a new integer programming (IP) formulation such that the roster resulting from solving the IP model is acceptable and can be used in practice at the hospital. In addition, this research contributes a matheuristic which 
decomposes the problem into subproblems. Such a method is necessary for tackling the most difficult instances for which standalone solvers were incapable of generating good results within acceptable computational runtimes. Results validated by the hospital managers provide insights that can help to facilitate the incorporation of constraints which are currently ignored into future nurse or physician rostering competitions.

\subsection{Cyclic rostering}

Cyclic workforce rostering, also referred to as rotating workforce rostering in the academic literature, generates, for each team, a fixed roster that repeats after a certain period [Musliu 2006]. The example introduced previously in Table 1 is considered cyclic if after the first week the first employee works the schedule of the second employee, the second employee works the schedule of the third, and so one. In this case, the cycle repeats after nine weeks.

These cycles mean that such rosters naturally enforce fairness between employees. However, while previously introduced cyclic models could not include complex constraints due to their model variables or the resulting lengthy computation times, the present research proposes a general model, based on the model defined in [Wickert et al. 2020], which is capable of capturing all the complex constraints required in practice.

The results obtained for the instances provided by the industry were better than those currently obtained by manual planners. Meanwhile, state-of-the-art results are achieved using the proposed model when solving the benchmark instances, outperforming existing methods based on heuristics, constraint programming, integer programming and satisfiability solvers [Wickert 2019].

\subsection{Nurse rerostering}

Uncertainty concerning employee availability is inherent in every personnel rostering problem. Common causes of employee absenteeism include heavy workloads, childcare obligations and illness [Forbes 2013]. Such absences may render an existing roster infeasible and difficult for human planners to remedy with last-minute changes. The fact that planners often find themselves under stress in such situations motivates the need to develop automated rerostering approaches which not only provide updated rosters very quickly but which also solve the problem more efficiently than humans.

Several novel strategies for rerostering based on relaxations of different problem parameters including soft constraints and the rescheduling horizon are investigated. For example, the difference in terms of solving time and solution quality is evaluated when the full scheduling horizon is considered and when only a limited part is taken into account. Existing literature had previously only investigated simplified scenarios considering single-skilled employees. We thus contribute a general integer programming formulation considering multi-skilled employees and various constraints often found in realworld problems. Moreover, while existing literature has mostly focused on metaheuristics approaches, computational experiments demonstrated that large instances, using both open-source and commercial solvers, can be solved to optimality with the proposed IP formulation [Wickert et al. 2019]. 


\subsection{Robust rostering}

Despite the proposed rerostering method being capable of repairing disrupted rosters, negative effects are unavoidable when using such a reactive method. This includes lastminute changes concerning employees' personal organization and high costs associated with the overtime required to remedy employee shortages. The generation of robust rosters is therefore crucial to minimize the occurrence of these negative factors. Academic literature, however, has lacked a metric for measuring the robustness of both single- and multi-skilled staff rosters.

This research contributes a metric which standardizes robustness quantification. Using the proposed metric, it is possible to quantify and enforce the robustness level of personnel rosters in advance. Moreover, operational costs associated with enforcing robustness in a roster are investigated. Computational experiments demonstrated that enforcing relatively low robustness levels typically resulted in the lowest final cost for both single- and multi-skilled employees. Higher robustness levels, meanwhile, resulted in higher initial and final costs since too many employees were unnecessarily assigned to reserve shifts [Wickert 2019].

\section{Final remarks}

This thesis combined scientific research with a practical application, harnessing the knowledge obtained through the $\mathrm{PhD}$ to meet the demands of society. The proposal of novel mathematical models are scientifically innovative and the papers describing these achievements are being welcomed by top-ranked international journals. In addition, applying the research to real-world scenarios shows that the research does not necessarily end when a paper is published. When it is possible to implement a useful product for public organizations based on the content expressed in a published paper, then it is clear that the research efforts were worthwhile and impacts positively the society.

\subsection{Registered software}

ProScheduleSolver - Solver for generating rosters, registered at National Institute of Industrial Property (INPI) number BR512019002293-9. The software is currently licensed to Hospital de Clínicas de Porto Alegre (HCPA).

The developed solution is adaptable and in use at HCPA since April of 2019. The benefits of using the software include: a reduction of time needed to generate roster, eliminating errors, decreased overtime concerning the scheduled professionals and a more homogeneous distribution of unpopular shifts.

An example of the adaptability of the proposed solution was to add HCPA's Block $\mathrm{B}$ to the roster, enabling the allocation of professionals who will treat Covid-19 patients. In this way, the scheduling of doctors who are treating Covid-19 cases at $\mathrm{HCPA}^{1}$ was carried out by ProScheduleSolver.

\subsection{Publications}

International peer-reviewed journals - Qualis A1:

\footnotetext{
${ }^{1} \mathrm{HCPA}$ is a reference for highly complex care for patients with Covid-19. The institution has already implemented ten new intensive care unit places in Block B and is preparing to open more vacancies. The goal is to achieve 105 new places before winter begins.
} 
- Wickert, Toni I.; Kummer Neto, Alberto F.; Boniatti, Márcio M.; Buriol, Luciana S.: An integer programming approach for the physician rostering problem, Annals of Operations Research, S.I.: PATAT 2018, 2020. DOI: https : / / doi •org/ $10.1007 /$ s10479-020-03552-5.

- Wickert, Toni I.; Smet, Pieter; Vanden Berghe, Greet: The nurse rerostering problem: strategies for reconstructing disrupted schedules, Computers and Operations Research, volume 104, pages 319-337, 2019. DOI: http://dx.doi.org/ $10.1016 / j . \operatorname{cor} .2018 .12 .014$;

International peer-reviewed conferences:

- Wickert, Toni I.; Kummer Neto, Alberto F.; Buriol, Luciana S.: An integer programming approach for the physician rostering problem, In Proceedings of the 12th International Conference of the Practice and Theory of Automated Timetabling (PATAT 2018), pages 53-67, Vienna - Austria, 28/08/2018 31/08/2018;

- Wickert, Toni I.; Sartori, Carlo; Buriol, Luciana S.: A Fix-and-Optimize VNS Algorithm Applied to the Nurse Rostering Problem, In proceedings of Sixth International Workshop on Model-based Metaheuristics (Matheuristic 2016), pages 1-12, Brussels - Belgium, 04/09/2016 - 07/09/2016.

Abstracts or extended abstracts presented in international conferences:

- Wickert, Toni I.; Mosquera Nuñez, Federico; Smet, Pieter; Thanos, Emmanouil: Optimizing city-wide vehicle allocation for car sharing, 32nd Annual Conference of the Belgian Operation Research Society (ORBEL 32), Liege, Belgium, 1/2/2018 - 2/2/2018;

- Wickert, Toni I.; Smet, Pieter; Vanden Berghe, Greet: A mathematical modeling approach for robust nurse rostering, 29th European Conference On Operational Research (EURO 2018), Valencia, Spain, 8/7/2018 - 11/7/2018;

- Wickert, Toni I.; Smet, Pieter; Vanden Berghe, Greet: Reconstructing Disrupted Nurse Rosters, Operational Research Applied to Health Services (ORAHS2017), Bath, United Kingdom, 30/7/2017 - 4/8/2017;

- Wickert, Toni I.; Sartori, Carlo; Buriol, Luciana S.: A fix-and-optimize heuristic applied to the Nurse Rostering Problem,Workshop on Applied Combinatorial Optimization Methods (WACOM 2016), Ouro Preto - Brazil, 21/03/2016 23/03/2016.

Publications in collaboration with undergraduate and master students:

- Portella, Victória; Buriol, Luciana S.; Wickert, Toni I.: Metaheurística Late Acceptance Hill Climbing Aplicada ao Problema de Escalonamento de Enfermagem, In Proceedings of the Brazilian Symposium on Operations Research (SBPO 2018), Rio de Janeiro, RJ, Brazil, 06/08/2018 - 09/08/2018;

- Meister, Tatiana; Wickert, Toni I.; Buriol, Luciana S.: Solving a physician rostering problem, IEEE Congress on Evolutionary Computation, Glasgow, United Kingdom, 9-24th July, 2020. (accepted paper). 
Seminars:

- Wickert, Toni I.: Strategies for reconstructing disrupted schedules. 2nd workshop on personnel planning and management. Place: Vrije Universiteit Brussel (VUB) - Room C2.07a (building C, 2nd floor) Date: 15/09/2017;

- Wickert, Toni I.: Reconstructing Disrupted Nurse Rosters. Place: KU Leuven Technologiecampus, Gebr. De Smetstraat 1, 9000 Gent. Date: 13/07/2017 11:00.

\section{References}

Burke, E. K., De Causmaecker, P., Vanden Berghe, G., and Van Landeghem, H. (2004). The state of the art of nurse rostering. Journal of scheduling, 7(6):441-499.

Erhard, M., Schoenfelder, J., Fügener, A., and Brunner, J. O. (2018). State of the art in physician scheduling. European Journal of Operational Research, 265(1):1 - 18.

Ernst, A., Jiang, H., Krishnamoorthy, M., and Sier, D. (2004). Staff scheduling and rostering: A review of applications, methods and models. European Journal of Operational Research, 153(1):3-27.

Forbes (2013). The causes and costs of absenteeism in the workplace. [Online; accessed 16-July-2018].

J. Bartholdi, J. (1981). A guaranteed-accuracy round-off algorithm for cyclic scheduling and set covering. Operations Research, 29:501-510.

Moz, M. and Pato, M. V. (2007). A genetic algorithm approach to a nurse rerostering problem. Computers \& Operations Research, 34(3):667 - 691. Logistics of Health Care ManagementPart Special Issue: Logistics of Health Care Management.

Musliu, N. (2006). Heuristic methods for automatic rotating workforce scheduling. International Journal of Computational Intelligence Research, 2(4):309-326.

Sohoni, M. G., Johnson, E. L., and Bailey, T. G. (2006). Operational airline reserve crew planning. Journal of Scheduling, 9(3):203-221.

Wickert, T. I. (2019). Personnel rostering : models and algorithms for scheduling, rescheduling and ensuring robustness. $\mathrm{PhD}$ thesis, Federal University of Rio Grande do Sul \& KU Leuven. [Available at: https://lume.ufrgs.br/handle/10183/203869].

Wickert, T. I., Boniatti, M. M., Kummer Neto, A. F., and Buriol, L. S. (2020). An integer programming approach for the physician rostering problem. Annals of Operations Research, SI: PATAT 2018.

Wickert, T. I., Smet, P., and Vanden Berghe, G. (2019). The nurse rerostering problem: Strategies for reconstructing disrupted schedules. Computers \& Operations Research, $104: 319-337$.

Xie, L. and Suhl, L. (2015). Cyclic and non-cyclic crew rostering problems in public bus transit. OR Spectrum, 37(1):99-136. 